\title{
Modelación del cloro residual y subproductos de la desinfección en un sector piloto del sistema de distribución de agua potable de la ciudad de Cali
}

\author{
Luís D. Sánchez"» , Sonia Rodríguez*, Juan C. Escobar**, Patricia Torres*** \\ *Instituto Cinara, Universidad del Valle \\ **Planta de Potabilización Puerto Mallarino, EMCALI EICE ESP \\ ***Escuela de Ingeniería de Recursos Naturales y del Ambiente, Universidad del Valle \\ §luisanc@univalle.edu.co
}

(Recibido: Abril 07 de 2010 - Aceptado: Junio 10 de 2010)

\begin{abstract}
Resumen
Este estudio se orientó a modelar el comportamiento del cloro residual y subproductos de la desinfección en un sector piloto del sistema de distribución de agua potable de la ciudad de Cali, con aproximadamente 7,000 usuarios (30,000 habitantes) abastecido con agua procedente del río Cauca. Los resultados indican que este tipo de modelación es una valiosa herramienta de trabajo para hacer seguimiento de la calidad del agua en la red a un mayor nivel de profundidad. En el sector piloto estudiado, el $80 \%$ del cloro residual estuvo por encima de $0.3 \mathrm{mg} / \mathrm{L}$. El subproducto de la desinfección predominante fue el cloroformo, representando el $95 \%$ de los trihalometanos, con valores de $80 \mu \mathrm{g} / \mathrm{L}$ en el $95 \%$ de los puntos de la red. El comportamiento del cloro residual y del cloroformo se vio afectado por la edad del agua en la tubería, la cual se incrementó cuando el sistema fue abastecido por el tanque de almacenamiento.
\end{abstract}

Palabras Claves: Cloroformo, Cloro residual, Edad del agua, Modelación calidad de agua, Trihalometanos.

\section{Modeling of residual chlorine and disinfection by-products of a pilot sector from the drinking water distribution system of Cali city}

\begin{abstract}
This study was designed to model the behavior of residual chlorine and disinfection by-products of a pilot sector of the drinking water distribution system in the city of Cali with about 7.000 users $(30.000$ inhabitants) supplied with water from the Cauca River. The results indicate that this type of modeling is a valuable tool for monitoring the water quality in the network at deeper levels. In the pilot study, $80 \%$ of residual chlorine was above $0.3 \mathrm{mg} / \mathrm{L}$. The predominant disinfection by-product was chloroform, representing $95 \%$ of trihalomethanes, with values of $80 \mathrm{mg} / \mathrm{L}$ in $95 \%$ of the points of the network. The behavior of residual chlorine and chloroform was affected by the water age in the pipe, which increases when the system was supplied by the storage tank.
\end{abstract}

Keywords: Chloroform, Residual chlorine, Water age, Water quality modeling, Trihalomethanes. 


\section{Introducción}

En un sistema de distribución de agua potable es esencial el suministro de agua segura a los consumidores; sin embargo, ésta puede sufrir variaciones por su naturaleza misma, por la extensión de la tubería, la existencia de tanques del almacenamiento, válvulas e hidrantes, así como reparaciones, infiltraciones por intermitencia y conexiones cruzadas. Para prevenir riesgos por alteraciones de la calidad del agua en la red, se deben analizar problemas como incremento de la edad del agua, decaimiento del cloro residual, control de la formación de los subproductos de la desinfección (SPD), recrecimiento microbiano, corrosión, incrustaciones y formación de biopelículas, entre otros (WHO,2004).

La pérdida del desinfectante residual es un proceso químico que da origen al problema más común de calidad de agua en una red que es la formación de SPD, la cual es función del tiempo y de su velocidad de desaparición, que puede estar afectada por aspectos como la actividad microbiológica, la temperatura, la nitrificación, la exposición a la luz ultravioleta y la cantidad y tipo de compuestos que generan demanda del desinfectante como compuestos orgánicos e inorgánicos (WHO, 2004). El cloro reacciona con la materia orgánica y forma SPD, incluyendo trihalometanos (THM) y ácidos halo-acéticos (AH), los cuales pueden ser cancerígenos y generar otros problemas a la salud (Boulos et al., 2006).

Para los THMs, existen cuatro definiciones básicas (Red Iberoamericana de Agua Potable,2003): THMs instantáneos: medidos al momento del muestreo; THMs finales: concentración final en el punto más alejado de la red principal; formación potencial de THMs: diferencia entre la concentración instantánea de THMs y los THMs finales y el potencial máximo total de THMs: concentración máxima de THMs bajo las condiciones más favorables para su formación. A su vez, los cuatro compuestos halogenados de mayor potencial de formación son: cloroformo $\left(\mathrm{CHCl}_{3}\right)$, bromodicloroformo, $\left(\mathrm{CHCl}_{2} \mathrm{Br}\right)$, dibromocloroformo, $\left(\mathrm{CHClBr}_{2}\right) \mathrm{y}$ bromoformo, $\left(\mathrm{CHBr}_{3}\right)$ (Sadiq \& Rodríguez, 2003)

Para el análisis de la calidad del agua en la red, los avances en la modelación hidráulica han facilitado la incorporación de conceptos para modelar su calidad; cuando los modelos hidráulicos son calibrados y validados. Estos modelos pueden predecir la variación de la concentración de sustancias disueltas en el tiempo, bajo un conjunto conocido de condiciones hidráulicas, y se basan en la desaparición del cloro; siendo el modelo cinético de decrecimiento de primer orden (en el cual la velocidad de reacción se expresa como una función potencial y la concentración limite (CL) tiende al decrecimiento de la sustancia $(\mathrm{CL}=0)$, cuyo exponente de reacción $\mathrm{n}$ es 1 y la constante de reacción $\mathrm{K}_{\mathrm{b}}<0$ ) es el más simple y utilizado tal como se muestra en la Ec. (1) (Vasconcelos et al., 1997; Vieira et al., 2004).

$$
\mathrm{C}=\mathrm{C}_{0} \exp \left(-\mathrm{k}_{\mathrm{b}} \mathrm{t}\right)
$$

Donde: $\mathrm{C}$ : concentración de $\mathrm{Cl}, \mathrm{C}_{\mathrm{o}}$ : concentración inicial $\mathrm{Cl}, \mathrm{K}_{\mathrm{b}}$ : constante $\mathrm{de}$ decaimiento y $\mathrm{t}$ : tiempo

Este modelo de decaimiento ha mostrado ser consistente para la mayoría de aplicaciones en sistemas de distribución (NCEL, 2004); pero, predecir el comportamiento del cloro a través de la red de distribución es complejo debido a la geometría de las tuberías, las condiciones dinámicas del flujo y la naturaleza variada de la reactividad del cloro (Walsky et al., 2001). Según (Rossman, 2000), largos tiempos de reacción producen generalmente altos consumos del desinfectante residual y mayor formación de SPD. Por otro lado, un aumento del $\mathrm{pH}$ incrementa los THM y reduce los AH y la temperatura tiene un efecto directo por el aumento de la velocidad de reacción (Rodríguez \& Sérodes, 2001).

El decaimiento del cloro se evalúa en la masa de agua y en la pared de la tubería (NCEL, 2004); para la masa de agua se aplica el modelo cinético de primer orden en que $\mathrm{K}_{\mathrm{b}}$ varía con $\mathrm{C}_{\mathrm{o}}$, lo que a su vez determina el número de sitios reactivos 
disponibles (o reactantes) (UKWIR, 1998; Powell et al., 2000). Cuando se tiene una alta $\mathrm{C}_{0}$, se retarda el decaimiento de $\mathrm{Cl}_{2}$ y se obtienen valores bajos de $\mathrm{K}_{\mathrm{b}}$ (EPA, 2003; Fang et al., 1999). Para determinar la demanda del cloro con respecto a la pared, éste se mide en los puntos de entrada y salida para una gran longitud de tuberías, garantizando que no ingresen caudales adicionales (Powell et al., 2000).

Los criterios de selección de puntos de muestreo para calibrar los modelos son diversos. Para cloro residual, se hacen mediciones en los tanques y puntos de inyección (Walsky et al., 2001); otros autores sugieren una variedad de 23 criterios con mediciones a lo largo de todo el sistema (Alcocer \& Tzatchkov, 2004) o medir en los extremos de la red con presiones bajas y en el punto inicial a la salida de los tanques (Xin \& Hong-bin, 2006). Para los THM, se hacen mediciones en zonas que registran altos tiempos de residencia y bajas concentraciones de cloro residual (Rodríguez \& Sérodes, 2001).

Para la modelación se requiere de una estructura matemática para combinar las reacciones que ocurren dentro de la red, teniendo en cuenta el transporte físico del desinfectante desde la masa de agua a la pared de la tubería y las reacciones químicas que ocurren en este proceso (EPA, 2006). Es así como bajo el principio de conservación de la masa, acoplado con las cinéticas de la reacción, el simulador EPANET (Walsky et al., 2001) modela la calidad del agua. Los fenómenos contemplados en ese modelo son el transporte conectivo en las tuberías, mezcla en la confluencia de las tuberías y en los depósitos de regulación y reacciones en la masa de agua y en las paredes de la tubería (EPA, 2006; Boulos et al., 1996).

Este trabajo se orientó a modelar el comportamiento del cloro residual y de SPD en un sector piloto del sistema de distribución de agua potable de la ciudad de Cali, abastecido con agua procedente del río Cauca.

\section{Metodología}

Inicialmente el sistema se modeló hidráulicamente y cuando estuvo debidamente calibrado (ajuste de los datos de entrada hasta que las salidas simuladas reflejen los datos de campo observados) y validado (resultados del modelo cercanos a los de campo cumpliendo con los criterios de precisión en un período de tiempo definido), se modeló la calidad del agua analizando el comportamiento de la edad del agua, el cloro residual y los SPD.

El software de modelación empleado fue EPANET (Walsky et al., 2001) el cual se seleccionó por las siguientes razones: el acceso al número de tramos permite considerar diferentes tipos de demanda en los nodos con su propia curva de modulación en el tiempo; simula el avance y destino final de las sustancias reactivas cuya concentración crece o decrece en el tiempo; permite simular el tiempo de permanencia del agua en la red y simula las reacciones que tienen lugar tanto en el seno del agua como en las paredes de las tuberías. Adicionalmente EPANET es una herramienta de dominio público sin costo y su utilización se ha generalizado en todo el mundo.

\subsection{Sector de estudio}

Se definió sobre la base, de que se pudiera aislar el análisis hidráulico y de calidad agua, de toda la red de Cali, a su vez fue un punto extremo de todo el sistema. El área de trabajo fue de $800 \mathrm{Ha}$, localizado en el sur-occidente de la ciudad de Cali. Abastece a un total de 17 barrios con una población de 30.000 habitantes, representando el 6,4\% del total de la red de la ciudad. La red tiene una longitud total de tubería de $91 \mathrm{Km}$, con diámetros menores a $150 \mathrm{~mm}(60 \%$ de los tubos) y material PVC (64\% de los tubos). Como el objetivo principal fue la calidad del agua, se modeló para la totalidad de los tramos, o sea, que no se hizo esqueletización de la red. Un esquema de la red y los puntos de control se presentan en la Figura 1.

\subsection{Datos de caudal}

Se ajustaron de la base de datos de suscriptores registrados en la oficina de facturación de EMCALI, EICE, ESP, que es la empresa prestadora de los servicios públicos de la ciudad de Cali; la herramienta utilizada para llevar a cabo el proceso de extracción de información fue el paquete aplicativo "Open Smart" que usa la oficina de facturación; las bases de datos se correlacionaron en el programa Office Access 2003, teniendo en cuenta el número de identificación del contador. 


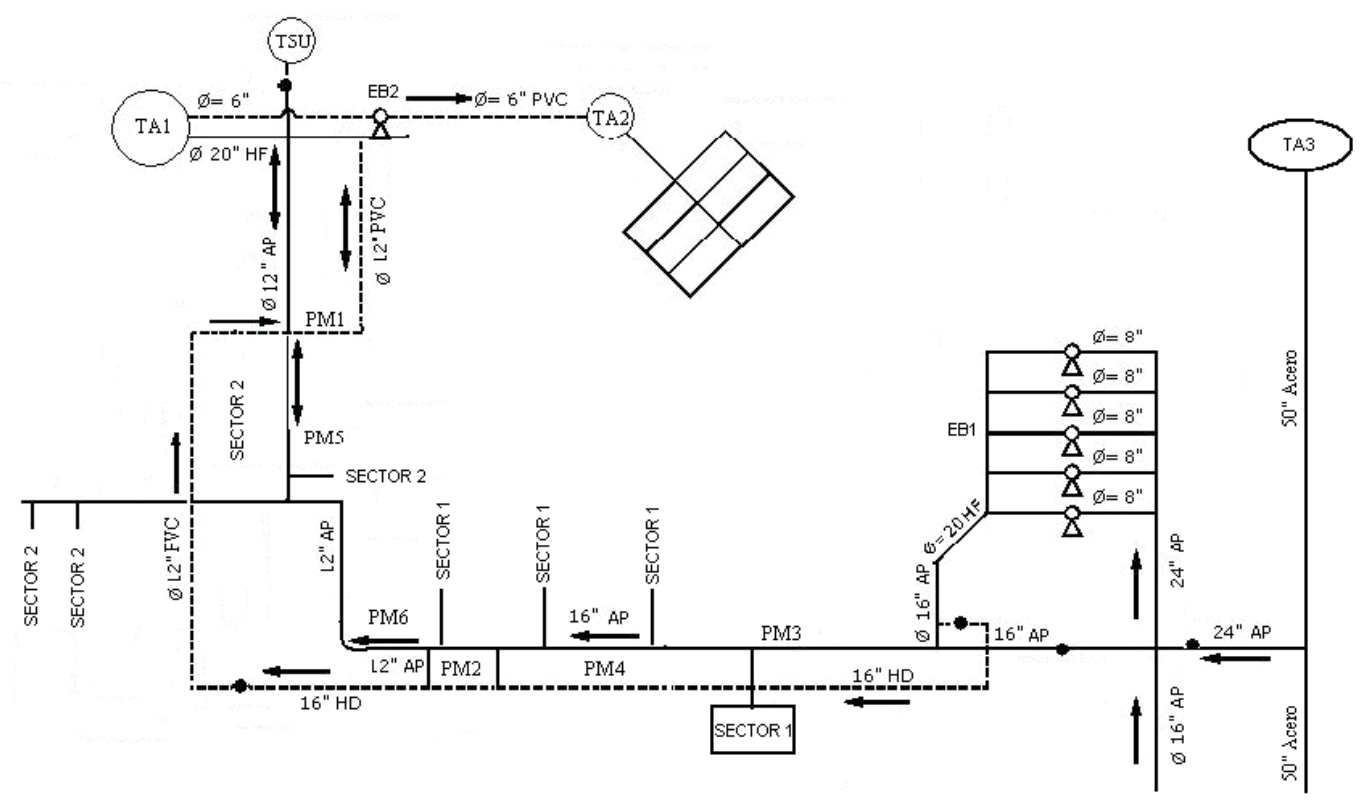

Figura 1. Esquema del sistema de distribución de agua y puntos de muestreo

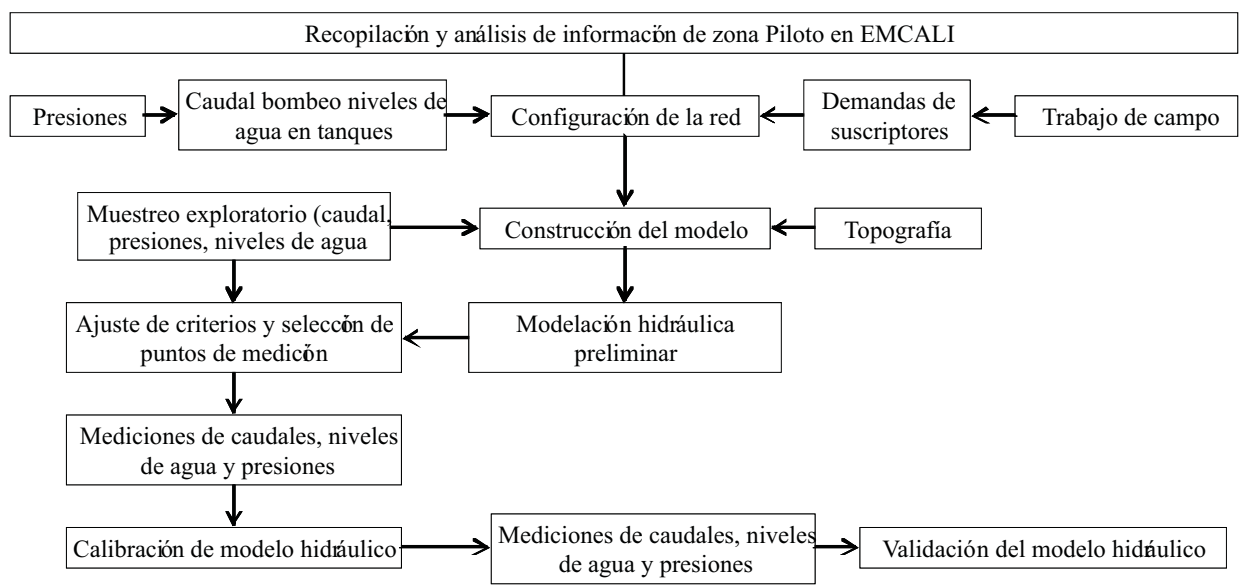

Figura 2. Recopilación y análisis de información en el sector piloto

Por medio de un sistema de información geográfica (GIS), se seleccionaron los suscriptores del sector de estudio y posteriormente se ubicaron, uno a uno, aquellos no geo-referenciados, así se ajustó el número de suscriptores. La Figura 2 presenta el procedimiento para la recopilación y análisis de información en el sector piloto.

\subsection{Puntos de medición}

Los puntos, los parámetros y los criterios para medición de presiones y caudales en la zona de estudio, se relacionan en la Tabla 1, esos valores se obtuvieron a partir del modelo hidráulico validado y fue el punto de partida para la modelación de la calidad del agua. 
Tabla 1. Puntos de medición, parámetros y criterios de selección.

\begin{tabular}{lll}
\hline Pto. & Parámetro & Criterio de selección \\
\hline EB1 & Caudal de bombeo & Punto de entrada al sistema. \\
TA1 & Niveles de agua & Tanque de compensación. \\
TA2 & Niveles de agua & Tanque de almacenamiento. \\
PM1 & Caudal-presión & Tubería troncal con más del $10 \%$ del caudal en el sistema. \\
PM2 & Caudal-presión & $\begin{array}{l}\text { Tubería troncal con más del } 10 \% \text { del caudal en el sistema: } \\
\text { tubería de impulsión. }\end{array}$ \\
PM3 & Caudal-presión & Tubería troncal con más del 10\% del caudal en el sistema. \\
PM4 & Caudal-presión & Tubería troncal con más del 10\% del caudal en el sistema. \\
PM5 & Caudal-presión & Tubería troncal con más del $10 \%$ del caudal en el sistema. \\
PM6 & Caudal-presión & Tubería troncal con más del 10\% del caudal en el sistema. \\
\hline
\end{tabular}

El coeficiente de decaimiento de cloro en la masa de agua se calculó por el método de la prueba de jarras (Lansey \& Boulos, 2005); el tiempo de duración de la prueba fue de 5 a 7 días dependiendo de la concentración inicial. El coeficiente de decaimiento del cloro en la pared de las tuberías se determinó con base en las mediciones de cloro realizadas en la red (Lansey \& Boulos, 2005), las cuales se realizaron en tres períodos para obtener una constante media representativa de la reacción del cloro con el agua. Se consideraron aquellos puntos de monitoreo que presentan una Co alta para que el cloro alcance un desarrollo total representativo para el ajuste de la curva exponencial (Alcocer et al., 2002).

El coeficiente de formación de cloroformo en la masa de agua se midió (en dos periodos de 10 y 14 días) a la salida de la EB1, ya que este punto es la entrada de agua al sistema y presenta la mayor concentración de cloro. Las muestras se tomaron a un flujo de $500 \mathrm{~mL} / \mathrm{min}$ en recipientes oscuros de 10 L y se distribuyó en recipientes ámbar de 500 $\mathrm{mL}$ destinados para el análisis de THM. Las muestras se analizaron dentro de los primeros 7 días después de la preservación. La formación potencial de THM se realizó por medio del procedimiento de saturación con cloro para analizar la concentración máxima en condiciones controladas de laboratorio durante 7 días.

\subsection{Calibración y validación hidráulica}

Consistió en el ajuste de los coeficientes de rugosidad y las demandas calculados para el sistema hasta que los niveles de agua en los tanques y las mediciones tomadas en los puntos de control se encontraran dentro de los niveles de precisión definidos. La Tabla 2 presenta los niveles alcanzados antes de iniciar la modelación de la calidad del agua.

\subsection{Edad del agua}

Se estimó partiendo de un tiempo de residencia en la EB1 igual a cero debido a que no se disponía de información de la edad del agua en el resto de la red. Se calculó para un período de 168 horas, cuando se observó su estabilización, según lo reportado por Vasconceles \& Boulos, 2005.

\subsection{Modelación de la calidad del agua}

En los diferentes puntos se tomaron muestras de cloro residual cada hora durante 24 horas por dos días. Los muestreos de calidad de agua se realizaron los días martes y miércoles y otro en un día de fin de semana (domingo). Los parámetros analizados se presentan en la Tabla 3. 
Tabla 2. Niveles de precisión para calibración y validación hidráulica

\begin{tabular}{|c|c|c|c|}
\hline \multirow{2}{*}{ Parámetro } & \multirow{2}{*}{ Niveles de precisión para calibración hidráulica } & \multicolumn{2}{|c|}{$\%$ Datos que cumplen } \\
\hline & & Calibración & Validación \\
\hline Caudales & $\begin{array}{l}\text { Los caudales en tuberías troncales modeladas } \\
\text { (donde el caudal }<10 \% \text { de la demanda total) deben } \\
\text { estar dentro de }+/-10 \% \text { de los caudales medidos. }\end{array}$ & 90 & 95 \\
\hline Presiones & $\begin{array}{l}\text { En el } 95 \% \text { de las mediciones de campo, las } \\
\text { diferencias de éstas con las modeladas, deben ser } \\
\text { inferiores a } 3,75 \mathrm{~m} \text { ( } 7,5 \% \text { de la pérdida máxima de } \\
\text { energía de un lado a otro del sistema). }\end{array}$ & 90 & 100 \\
\hline $\begin{array}{l}\text { Niveles de agua } \\
\text { en tanques }\end{array}$ & $\begin{array}{l}\text { La diferencia entre los niveles de agua observados } \\
\text { en tanques y los modelados debe ser }<0,30 \mathrm{~m} \text {. }\end{array}$ & 100 & 100 \\
\hline
\end{tabular}

Tabla 3. Parámetros de calidad del agua en los puntos de muestreo

\begin{tabular}{llcc}
\hline \multicolumn{1}{c}{ Parámetro } & \multicolumn{1}{c}{ Método de análisis } & Límite de detección & Precisión \\
\hline Cloro Residual $-\mathrm{Cl}_{2}$ & $\begin{array}{l}\text { DPD Titulo métrico } \\
\text { 4.500-Cl-F* }\end{array}$ & $0.10 \mathrm{mg} / \mathrm{L}$ & $0.01 \mathrm{mg} / \mathrm{L}$ \\
THM Totales & $\begin{array}{l}\text { Cromatografía de gases con } \\
\text { detección de captura de } \\
\text { electrones }\end{array}$ & $4.4 \mathrm{a} 14.8 \mathrm{ppb}$ & $6.0 \mathrm{ppb}$ \\
Cloroformo $-\mathrm{CHCl}_{3}$ & & \\
\hline
\end{tabular}

Para hacer la determinación se siguieron los siguientes pasos: $i$. Preparación de la solución tampón y solución dosificadora de cloro; ii. Determinación de la demanda de cloro verificando que la demanda de cloro residual después de 7 días no fuera superior a $1 \mathrm{mg} / \mathrm{L}$; iii. Aumento de la concentración de cloro para generar la máxima formación de THM en la muestra, manteniendo el $\mathrm{pH}$ en 7 unidades y $i v$. Titulación de la muestra por el método de DPD después de 7 días de realizada la cloración.

\section{Resultados y discusión}

El modelo de calidad de agua se calibró para un tiempo de modelación de 72 horas, con estabilización después de 48 horas. Se trabajó con $\mathrm{C}_{\mathrm{o}} 0.90 \mathrm{mg} / \mathrm{L}$ y $\mathrm{K}_{\mathrm{b}}$ en la masa de agua $-0.23 /$ día, muy próximo a lo reportado por (Lansey \& Boulos, 2005). El K $\mathrm{b}_{\mathrm{b}}$ en las paredes fue -0.10/día al comienzo del proceso; el coeficiente de formación de THM se ajustó en cada nodo donde se hicieron las mediciones hasta que se aproximara a los valores reales. La calibración se realizó ajustando $\operatorname{los} \mathrm{K}_{\mathrm{b}}$ hasta que las mediciones del cloro residual se acercaran a las calculadas por el modelo con una diferencia de $0.2 \mathrm{mg} / \mathrm{L}$. El nivel de precisión para la calibración del cloroformo se estableció en $6 \mu \mathrm{g} / \mathrm{L}$ por encima o por debajo del valor reportado y si la diferencia entre los valores afectados y el calculado por el modelo era menor del $10 \%$ del resultado reportado, se consideraba el modelo calibrado.

\subsection{Coeficiente de decaimiento del cloro en la masa de agua}

El comportamiento del cloro siguió el modelo de la Ec. (2), indicando tendencia a un modelo exponencial de decaimiento de primer orden. El $\mathrm{K}_{\mathrm{b}}$ para las pruebas realizadas varió entre -0.23/ día y -0.26/día, con un $\mathrm{R}^{2}$ de 0.98 . 


$$
\mathrm{Cl}=0.5481 \exp (-0.23 \mathrm{t})
$$

Donde: $\mathrm{t}=$ tiempo en días y $\mathrm{Cl}=$ concentración de cloro en $\mathrm{mg} / \mathrm{L}$

La variación de $\mathrm{Kb}$ se pudo deber a factores como la temperatura, el contenido de Carbono Orgánico Total (COT) (promedio $2.8 \mathrm{mg} / \mathrm{L}$ ), de Sólidos Disueltos Totales (SDT) (promedio $1.1 \mathrm{mg} / \mathrm{L}$ ) y la concentración inicial de cloro, por lo que Kb puede ser específico para cada fuente y red de distribución. Los valores encontrados están acordes a lo reportado por (Vasconcelos et al., 1997; EPA, 2003; EPA, 2006; Boulos et al., 1996) para reacciones de primer orden.

\subsection{Coeficiente de formación de cloroformo en la masa de agua}

Sobre la base de las mediciones de campo se encontró que a los 5 días de iniciadas las mediciones, el cloroformo dejó de aumentar y se estabilizó entre 100 y $108.6 \mu \mathrm{g} / \mathrm{L}$. Mediante la Ec. (3) se obtuvo el ajuste para el cálculo del coeficiente de formación del cloroformo, el cual se calculó para un período inicial de 2.7 días, obteniéndose un valor de $\mathrm{Kb}$ de 0.147 día. Esto significa que la tasa de formación de cloroformo fue de $36 \mu \mathrm{g} / \mathrm{mg} \mathrm{Cl}_{2}$, valor que se encuentra dentro del intervalo de 31 a $42 \mu \mathrm{g}$ TTHM/mg $\mathrm{Cl}_{2}$ reportado por (Gang et al., 2003).

$$
\mathrm{CHCl}_{3}=65.74 \exp (0.147 \mathrm{t})
$$

Donde: $\mathrm{t}=$ tiempo en días y $\mathrm{CHCl}_{3}=$ Concentración de cloroformo en $\mu \mathrm{g} / \mathrm{L}$

\subsection{Potencial de formación de trihalometanos (PFTHM)}

Para la obtención del potencial de formación de THM fue necesario conocer los THM iniciales y finales al consumirse el cloro a los 7 días. Los valores de PFTHM se encontraron entre 35-55 $\mu \mathrm{g} / \mathrm{L}$, valores inferiores a los encontrados en las plantas de tratamiento de la ciudad de Bogotá, Tibitoc, según (Marín, 2005).

\subsection{Edad del agua}

La Figura 3 presenta el comportamiento de la edad del agua en los puntos de medición (PM6, PM2, PM4 y PM3) y el caudal contra el tiempo, en sectores abastecidos directamente por la EB1. La edad del agua varió entre 2 y 5 horas, presentándose las mayores edades del agua entre las 0:00 y 6:00 horas del día, coincidiendo con los caudales bajos en la red y cuando los consumos fueron mínimos. Estos resultados guardan armonía con lo reportado por (EPA, 2002). Las menores edades del agua, coincidieron con los momentos de altos consumos, cuando los usuarios están demandando más agua del sistema.

En el sector abastecido por el TA1 y la EB2 (ver figura 1) simultáneamente, la edad del agua (en los nodos de seguimiento en la red 517, 375, 386, 65 y T8) varió entre 3 y 70 horas. Según la Figura 4, se observa que cuando el nivel de agua en el tanque está aumentando, el sector se abastece directamente por la EB2. Para esa situación las edades de agua fluctúan entre 3 y 4 horas.

Sin embargo, cuando en el TA1 empieza a descender el nivel del agua, el sector está siendo abastecido por él y las edades del agua se incrementan a valores entre 60 y 70 horas. Por lo anterior, el agua almacenada en el tanque contribuye al incremento en la edad del agua.

En el $83 \%$ de los tramos de las tuberías que conforman la red, la edad del agua fue inferior a 24 horas, indicando un buen comportamiento hidráulico del sistema. Las mayores edades de agua en el sistema se debieron al agua almacenada en el TA1; el envejecimiento del agua se presentó en el 17\% de las tuberías, cuando la edad del agua alcanzó 70 horas. Estos resultados están acordes a lo reportado en la evaluación de 800 sistemas de distribución en Estados Unidos (EPA, 2002), con edades máximas de 72 horas.

\subsection{Comportamiento del cloro residual}

La modelación del cloro residual se analizó teniendo en cuenta si el suministro de agua era solo de la EB o de ésta y del TA1. El cloro residual se relaciona con la edad del agua porque su decaimiento depende del tiempo que permanezca 


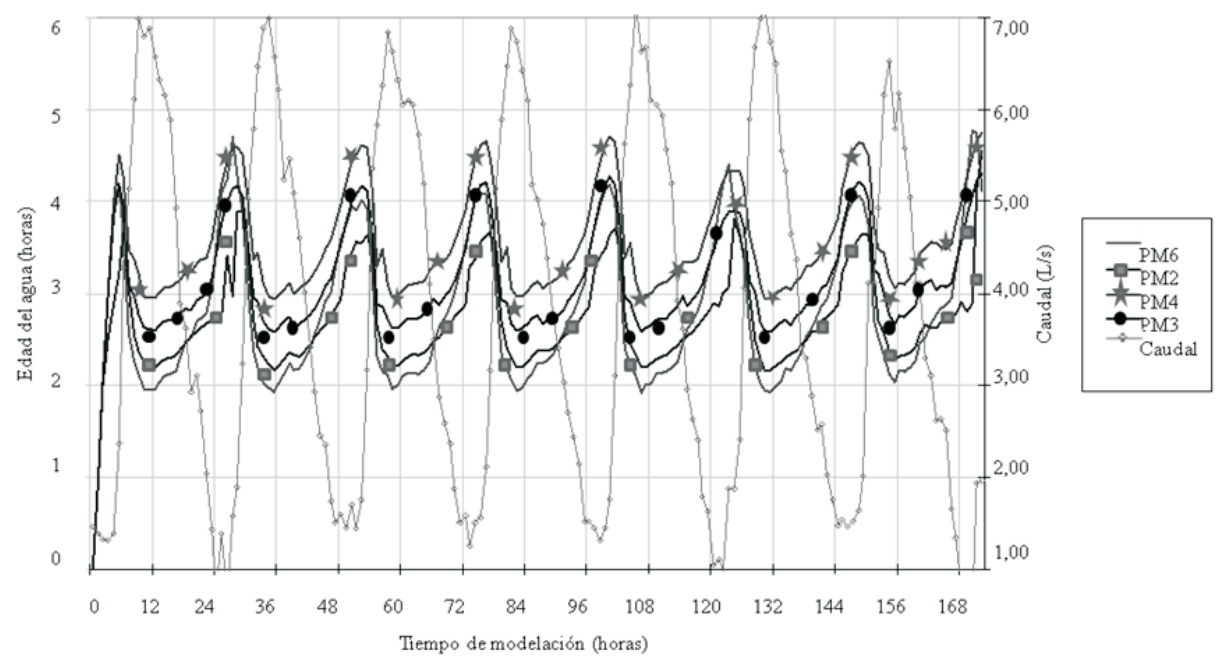

Figura 3. Relación edad del agua y la demanda (puntos PM6, PM2, PM3 y PM4, ver Tabla 1)

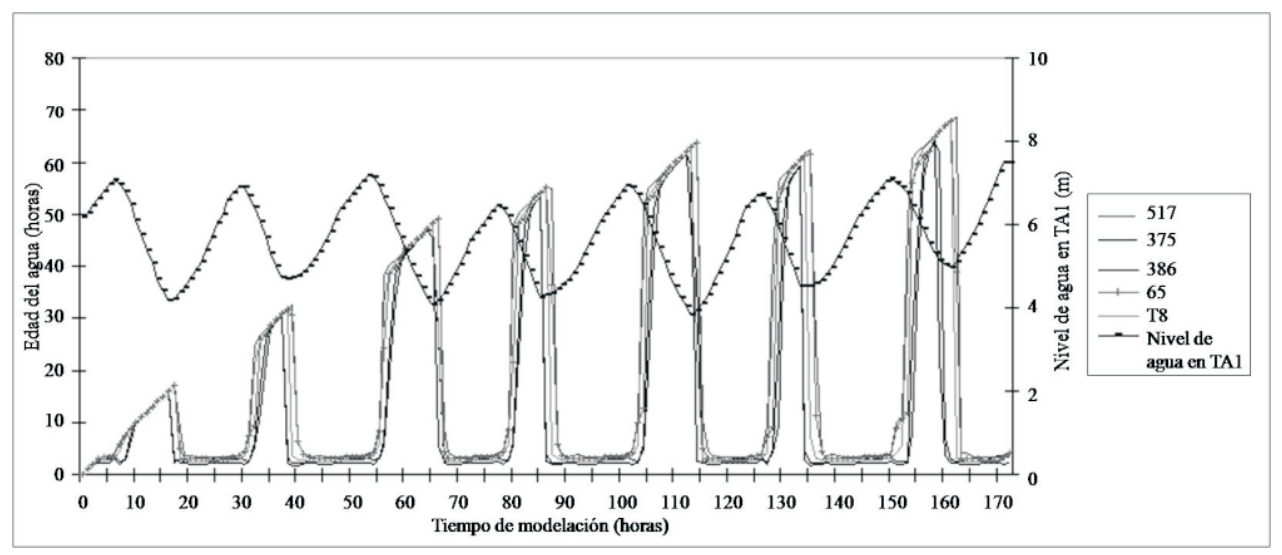

Figura 4. Relación edad del agua en los sectores 1, 2 y nivel de agua en el TA1 (517, 317, 386 y 65 nodos de control en la red)

en contacto con las diferentes sustancias presentes en el agua.

En la Figura 5 se aprecia que para los sectores alimentados directamente por la EB, el cloro residual varió entre 0,3 y $0,7 \mathrm{mg} / \mathrm{L}$, presentándose las menores concentraciones entre las 0:00 horas y las 6:00 am y las mayores entre las 6 am y las $6 \mathrm{pm}$. Las concentraciones más bajas se presentaron cuando la edad del agua fue alta.

La Figura 6 presenta un comportamiento diferente del cloro residual, especialmente en las zonas abastecidas por la EB2 y el TA1 simultáneamente; ésta corresponde a la modelación de los nodos de seguimiento del sector 2 (T517, T375, T386, T65, T8), donde el cloro residual varió entre 0.20 y $0.70 \mathrm{mg} / \mathrm{L}$, con los valores más altos entre las 0:00 horas y las 6:00 am. Cuando el sistema es abastecido por la EB2, presenta la menor edad del agua. Para ese sector, los bajos valores de cloro residual se presentan entre las 6:00 am y las 6:00 pm, cuando el sistema fue abastecido por el TA1, el cual contribuyó a que se presentaran mayores edades del agua. 


\subsection{Comportamiento de los THMs}

De las especies de THMs analizadas en el estudio, el cloroformo fue el THM predominante y correspondió al 95\% de los THM totales, seguido por el bromodiclorometano $\left(\mathrm{CHCl}_{2} \mathrm{Br}\right)$ con una concentración promedia de $4.1 \mu \mathrm{g} / \mathrm{L}$, acorde a lo reportado por (Vasconcelos et al., 1997). El cloroformo se modeló de la misma forma como se hizo para el cloro residual. La Figura 7 relaciona la edad del agua con el cloroformo para un tiempo de modelación de 168 horas, en una zona abastecida únicamente por la EB1, obteniéndose una edad del agua entre 2 y 4.5 horas. Se observó poca variación en la concentración del cloroformo y se tuvo baja disminución, cuando la edad del agua fue baja. Pero se tuvo un leve aumento cuando la edad del agua alcanzó las 4.5 horas. Esos leves aumentos pueden estar asociados a un mayor tiempo de contacto entre el cloro y la materia orgánica, con mayor posibilidad de formación de los THMs.

La edad del agua en las tuberías influencia directamente la concentración del cloroformo, lo que se pudo evidenciar en el sector 2 abastecido por la EB2 y el TA1, como se muestra en la Figura 8 , que relaciona la edad del agua con las variaciones en la concentración de cloroformo para un tiempo de modelación de 168 horas y se pudo apreciar que las mayores concentraciones de cloroformo se presentan cuando se incrementa la edad del agua en el sistema y disminuye cuando la edad del agua baja. La concentración de cloroformo se incrementó en $20 \mu \mathrm{g} / \mathrm{L}$ por efecto del aumento en la edad del agua, lo cual favorece un mayor tiempo de contacto del cloro con la materia orgánica presente en el agua.

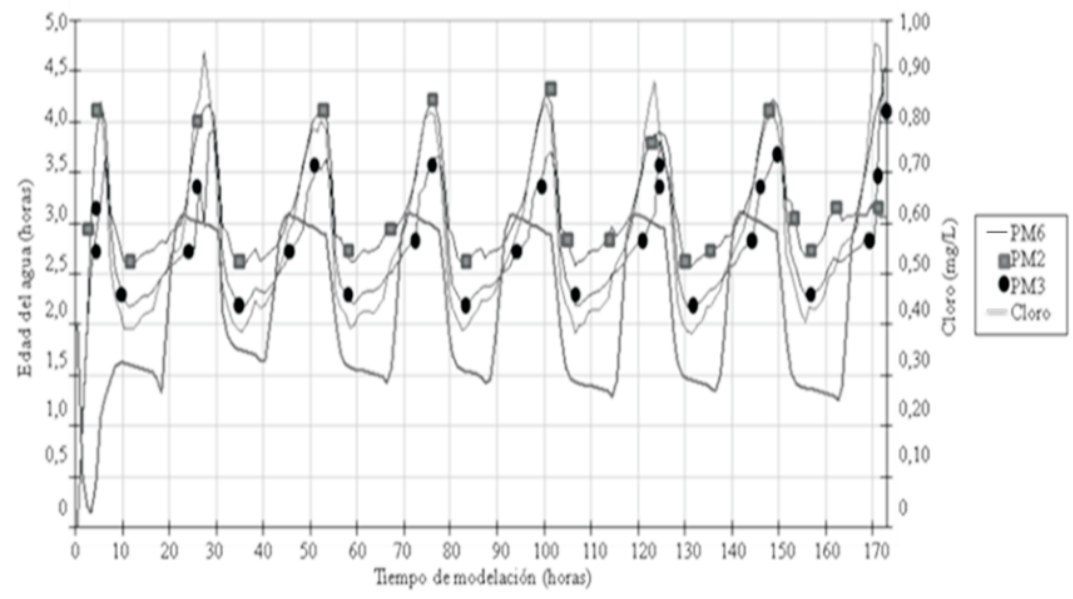

Figura 5. Cloro residual en el sector 1(puntos PM6, PM2, PM3, ver Tabla 1)

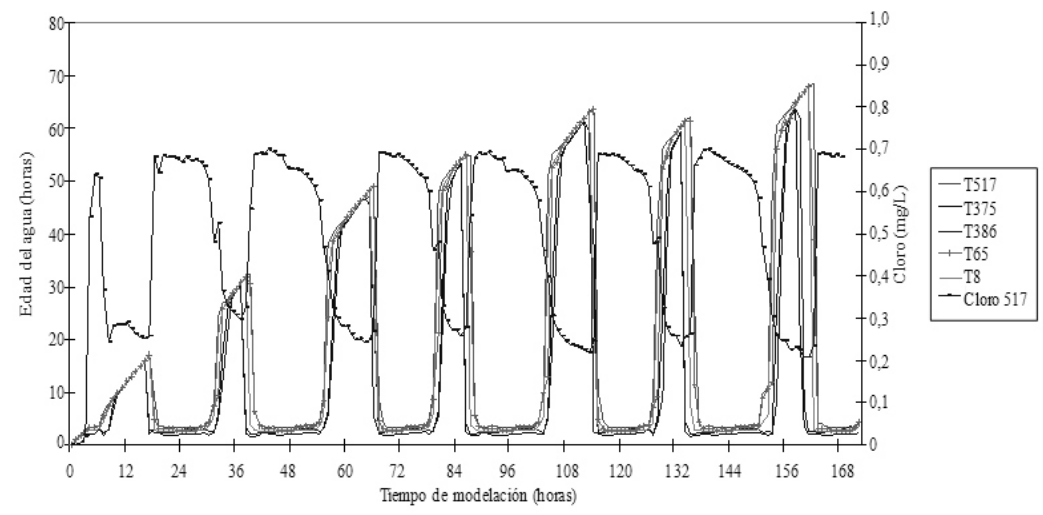

Figura 6. Relación edad del agua y cloro residual en el sector 2 (517, 317, 386 y 65 nodos de control en la red) 


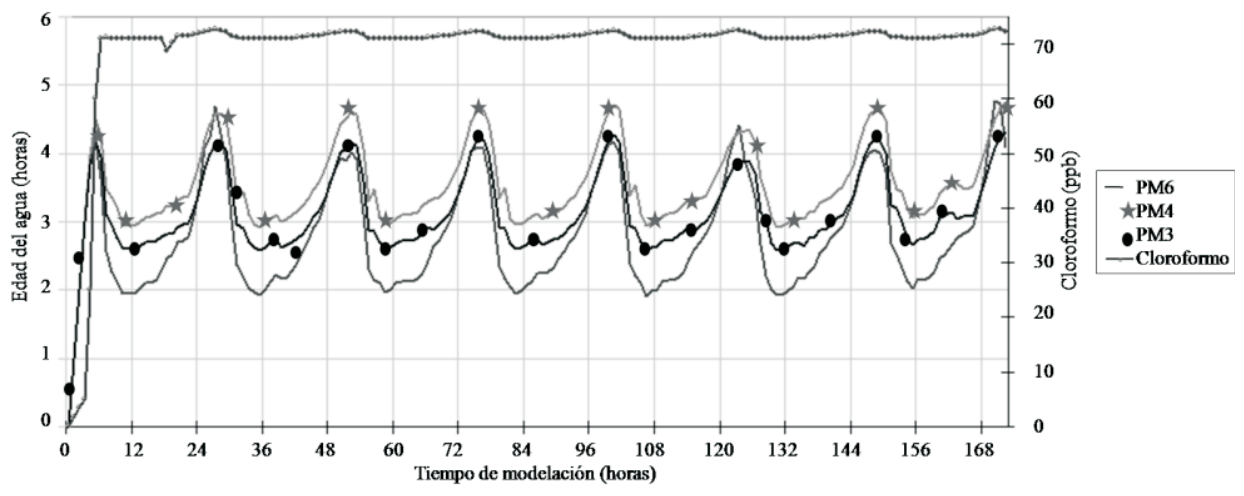

Figura 7. Relación de la edad del agua y el cloroformo en el sector 1(puntos PM6, PM2, PM3, ver Tabla 1)

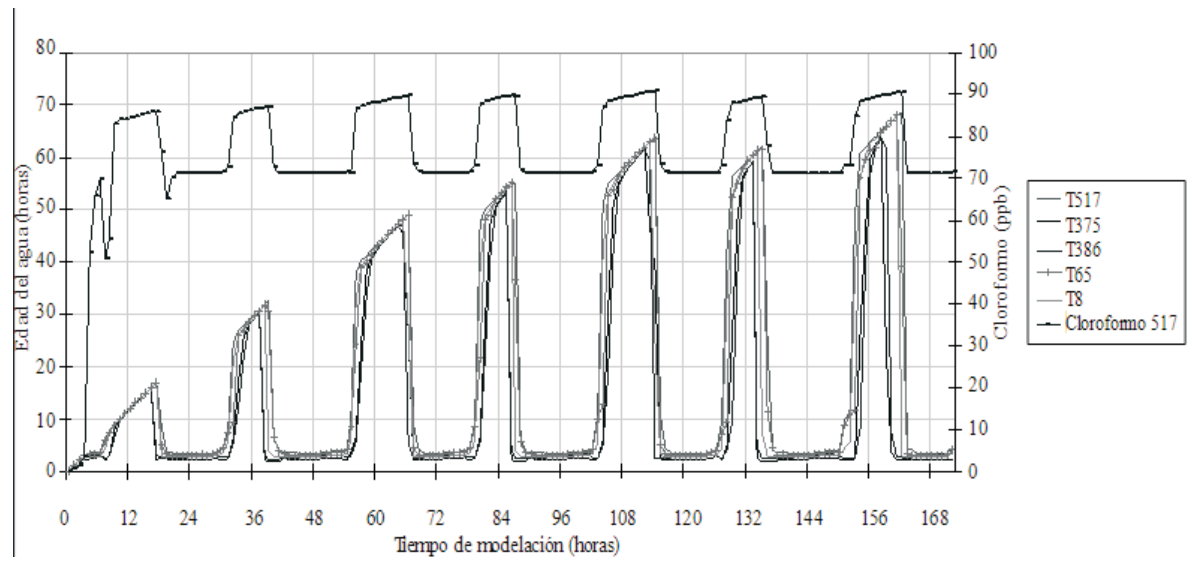

Figura 8. Relación edad del agua y cloroformo en el sector 2. (517, 317, 386 y 65 nodos de control en la red)

La modelación de la calidad del agua, en el sector de estudio, mostró que el $80 \%$ de las tuberías presentaron concentraciones de cloro residual por encima de $0.30 \mathrm{mg} / \mathrm{L}$, que es el valor guía mínimo establecido en (MPS y MAVDT, 2007) y el otro $20 \%$ requieren el ajuste respectivo por parte de la empresa prestadora del servicio de agua potable. Los puntos con bajo valor de cloro residual se encuentran en los sectores con edades de agua mayores a 24 horas y con velocidades de flujo menores a $0.10 \mathrm{~m} / \mathrm{s}$. Por otra parte el $95 \%$ de las tuberías presentaron concentraciones de cloroformo por debajo de $80 \mu \mathrm{g} / \mathrm{L}$, es decir que con relación a (WHO, 2004), la totalidad de los datos según este estudio están por debajo del valor guía de $200 \mu \mathrm{g} / \mathrm{L}$. Al considerar la normatividad colombiana, para THM totales, (MPS y MAVDT, 2007), se obtuvo que el $100 \%$ de los datos estuvieron por debajo de $200 \mu \mathrm{g} / \mathrm{L}$ y en consecuencia en el sector modelado, el riesgo por subproductos de la desinfección fue bajo.

\section{Conclusiones}

- La modelación del cloro residual y subproductos de la desinfección es una valiosa herramienta de trabajo para hacer seguimiento de la calidad del agua en la red a un mayor nivel de profundidad. En el sector piloto evaluado, el 80\% del cloro residual en la red estuvo por encima de $0.3 \mathrm{mg} / \mathrm{L}$, siendo necesario revisar, ajustar y hacer seguimiento al comportamiento del cloro residual 
en el $20 \%$ restante para garantizar el aseguramiento de la calidad del agua a todos los usuarios.

- $\quad$ El 95\% de los THMs fue el cloroformo, presente en el $95 \%$ de las tuberías con un valor inferior a $80 \mu \mathrm{g} / \mathrm{L}$, menor al límite establecido en las guías de la OMS.

- La edad del agua en la tubería se incrementa cuando el sistema es abastecido por el tanque de almacenamiento, afectando el comportamiento del cloro residual y el cloroformo en la red.

- Para EMCALI EICE ESP, es clave revisar el criterio de diámetro mínimo de las tuberías, el funcionamiento de tramos con flujo discontinuo, disminuir el número de puntos muertos e incrementar el cloro residual en los puntos con valores inferiores a la norma.

\section{Agradecimientos}

Los autores agradecen a EMCALI EICE ESP y la Universidad del Valle, la financiación y todo el soporte recibido para desarrollar este trabajo. Al ingeniero Luis Germán Delgado por sus aportes en el entendimiento de la operación del sistema y al equipo de trabajo de los grupos de investigación Abastecimiento de Agua del Instituto Cinara y Estudio y Control de la Contaminación Ambiental de la Escuela EIDENAR.

\section{Referencias bibliográficas}

Alcocer V., Tzatchkov, V., Felician,o D., Mejía, E., \& Martínez, E.(2002). Implementación y calibración de un modelo de calidad del agua en sistemas de agua potable. pp.219. Informe Final, IMTA-CNA, Jiutepec, Morelos.

Alcocer, V., Tzatchkov, V., \& Arreguín, F. (2004). Modelo de Calidad del Agua en Redes de Distribución. Ingeniería Hidráulica en México. 19 (2), 77-88.

Boulos, P., Lansey, K., \&Karney, B. (2006). Comprehensive water distribution systems analysis handbook for engineers and planners. 6:1-120.Ed.1. MWH SOFT Pasadena, California USA.
Boulos, P.F., Mau, R., \& Bowcock, R.(1996). Modeling distribution storage water quality: an analytical approach. Journal of Applied Mathematical Modelling. 20 (4), 329-338.

EPA 815-D-03-004. (2003). Stage 2 Disinfectants and Disinfection Byproducts Rule Significant Excursion Guidance Manual.2-3.

EPA. (2006). Stage 2 Disinfectants and Disinfection by Products Rule Chapter 6. http://www.epa.gov/safewater/disinfection/stage2 /compliance.html.

EPA. (4601M) (2002). Effects of water age on distribution system water quality. Distribution system issue paper. 5-6 Washington DC.USA.

Fang, H., West, J.R., Barker, R.A., \& Foster, C.F. (1999). Modelling of chlorine decay in municipal water supplies. 2735-2746, Water Research. 33(12), 2735-2746.

Gang, D., Clevenger ,T., \& Banerji, S.(2003). Relationship of chlorine decay and THMs formation to NOM size. Journal of hazardous materials. 96(1), 1-12.

Lansey, K., \& Boulos, P. (2005). Comprehensive Handbook on water quality analysis for distribution system. Ed. 2. 4:1-86. MWH SOFT Pasadena, California USA.

Marín, C. (2005). Evaluación de la formación potencial de trihalometanos en agua potable usando micro extracción en fase sólida como método de extracción. Universidad de los Andes. http://columbus.uniandes.edu.co:5050/dspace/bits tream/1992/475/1/mi_816.pdf.

Mays, L. (2003). Manual de Sistemas de Distribución de Agua. pp. 350-380 Ed. Mc. Graw Hill, Ed.1. Madrid.España.

Ministerios de la protección social, y vivienda y ambiente, vivienda y desarrollo territorial. Resolución 2115 de 2007. Instrumentos básicos y frecuencias del sistema de control y vigilancia para la calidad del agua para consumo humano. Colombia.pp 3. 
NCEL, National Civil Engineering Laboratory .(2004), 1700-066 Av. Brasil 101, Lisboa, Portugal, 454-456.

Powell, J., Hallam, N., West, J., Forster, C., \& Simas, J. (2000). Factors which control bulk chlorine decay rates. 117-126 Water Research, 34(1) 117-126.

Red Iberoamericana de Agua Potable (2003) Consideraciones sobre los Subproductos de la Desinfección. Capitulo 14. (Sofía Esperanza $\mathrm{G}$ a $\mathrm{r}$ r i d o H o y o s ). http://tierra.rediris.es/hidrored/ebooks/ripda/\#.

Rodríguez, M. \& Sérodes, J.B. (2001) Spatial and temporal evolution of trihalomethanes in three water distribution systems. 1572-1586. Water Research.35(6), 1572-1586.

Rossman, L. (2000) EPANET users manual. US Environmental Protection Agency, National Risk Management Research Laboratory, 21-186 Office of Research and Development, Cincinnati, $\mathrm{OH}$.

Sadiq,R. \& Rodriguez,M.J.(2003). Disinfection by-products (DBPs) in drinking water and predictive models for their occurrence: a review. Science of the total environment. 321 (1-3), 21-46.

UKWIR.UK Water Industry Research. (1998). Chlorine and Monochloramine Demand of Materials For Drinking Water Distribution Systems. Report 98/DW/03/9, London.
Vasconceles, J.J., Boulos, P.F. (1996). Characteristics and modelling of chlorine decay in distribution systems. American Water Works Association and American Water Works Association Research Foundation, (AWWA) pp.115 -164. Cincinnati USA.

Vasconcelos, J., Rossman, L.A., Grayman, W.M., \& Boulos, P.F., Clark, R.M.(1997) Kinetics of Chlorine Decay. Journal American Water Works Association. 89 (7), 54-65.

Vieira P., Coelho, S. T. \& Loureiro, D. (2004). Accounting for the influence of initial chlorine concentration, TOC, iron and temperature when modelling chlorine decay in water supply. Journal of water supply: research and technology. AQUA. 53(7), pp. 454-456

Walsky, T., Chase, D., \& Savic, D. (2001). Water Distribution Modeling. 56-60, Ed.Haestad Press. Waterbury. USA.1ed.

WHO(2004). Guidelines for Drinking Water Quality. World Health Organization, Ed. WHO library, 3 ed. pp 62-63

Xin,L., \& Hong-bin,Z.(2006). Development of a Model for Predicting Trihalomethanes Propagation in Water Distribution Systems. Chemosphere. 62 (6),1028-1032. 